\title{
Prevalence of epilepsy in prisoners: systematic review
}

\author{
Seena Fazel, Evangelos Vassos, John Danesh
}

Henry Maudsley, doyen of 19th century British psychiatry, believed that people with epilepsy were particularly prone to violence and criminality, ${ }^{1}$ a view shared by many leading psychiatrists and neurologists today. ${ }^{2}$ Epilepsy is typically claimed to be about two to four times more common in prisoners than in the general population, but the epidemiological evidence cited to support this claim is of uncertain validity. Previous surveys of prisoners have involved unrepresentative populations, proxy measures (such as use of anticonvulsant drugs), and secondhand respondents (such as prison medical officers). To help clarify the evidence, we conducted a meta-analysis of available surveys based on personal clinical interviews in general prison populations.

\section{Methods and results}

We sought studies of the prevalence of epilepsy, fits, convulsion, or seizures in approximately general prison populations (that is, excluding studies of prisoners referred for neuropsychiatric assessment) reported between January 1966 and August 2001 by computer based searches (Embase, PsycINFO, Medline), scanning of relevant reference lists, and hand searching of forensic psychiatry journals and other relevant journals. We used combinations of keywords relating to epilepsy (for example, epilep*, seizure, fit, convulsion) and to prisoners (for example, inmate, sentenced, remand, detainee, felon). Eligible studies reported on an adult history of chronic epilepsy (defined as a condition characterised by two or more recurrent seizures, unprovoked by any immediately identifiable cause). ${ }^{\mathrm{w}-\mathrm{w} 7}$ Two investigators independently extracted the following data according to a fixed protocol: geographical location, year of survey, number of prisoners, sampling strategy, response rate, diagnostic criteria, mean age and sex of prisoners, and number of prisoners reporting a history of epilepsy in adult life. Discrepancies were resolved by discussion and by correspondence with authors of surveys. Calculation of confidence intervals and data synthesis involved standard methods, as previously described. ${ }^{3}$

We identified seven relevant surveys (3111 prisoners). ${ }^{\mathrm{w}-\mathrm{w} 7}$ Reported sampling strategies included complete sampling of entire prisons (584 prisoners), ${ }^{\mathrm{w}, \mathrm{w} 7}$ stratified random sampling (2027), ${ }^{\mathrm{w} 1, \mathrm{w4}}$ and inclusion of consecutive prisoners (500). ${ }^{\mathrm{w} 3, w 5, w 6} \mathrm{Six}$ studies reported response rates in excess of $90 \%$, w1-w6 and one study reported a response in excess of $75 \%$. ${ }^{\mathrm{w} 7}$ All studies were based on clinical interviews (none was supplemented by neurological examination or other medical investigation). All respondents were sentenced inmates, the weighted mean age was 29 years, $90 \%$ were men, and $22 \%$ had been convicted of violent offences. Three surveys were conducted in the United Kingdom (2297 prisoners), ${ }^{\mathrm{w} 1, \mathrm{w} 3, \mathrm{w} 4}$ two in the United States (591), ${ }^{\mathrm{w} 2, \mathrm{w} 6}$ and one each in Canada $(100)^{\mathrm{w} 5}$ and Nigeria (93). ${ }^{\mathrm{w} 7}$ Overall, 23 of the prisoners in these surveys reported a history of chronic epilepsy, yielding a

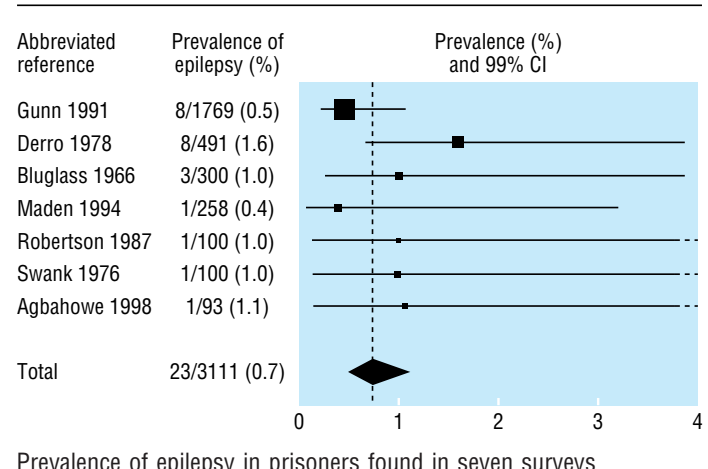

Prevalence of epilepsy in prisoners found in seven surveys

prevalence rate of $0.7 \%$ (95\% confidence interval $0.5 \%$ to $1.1 \%$ (figure), and we found no significant heterogeneity among the seven surveys $\left(\chi_{6}^{2}=8.3\right.$; $\mathrm{P}>0.10)$.

\section{Comment}

In contrast with claims widely published in standard texts and other sources, ${ }^{2}$ this synthesis of seven surveys involving more than 3000 participants in general prison populations indicates that only about $1 \%$ reported a history of chronic epilepsy. The prevalence rate in general populations is also approximately $1 \%$ for men aged 25-35 years, according to community based surveys that used definitions of epilepsy most comparable to those used in the studies reviewed here. ${ }^{45}$ Any publication bias in the reports contributing to this meta-analysis would probably tend to exaggerate the prevalence rates of epilepsy among prisoners, which reinforces our conclusion that the available epidemiological evidence provides no good support for the alleged link between epilepsy and criminality.

The following investigators provided additional data: $\mathrm{S}$ Agbahowe, G Hannon, N Singleton, C Taylor. C Meux commented helpfully. P Appleby plotted the figure.

Contributors: All of the authors were involved in the design, conduct, analysis, and interpretation of the study. SF will act as guarantor.

Funding: SF was supported by a grant from the Wellcome Trust. JD was supported by the Raymond and Beverly Sackler Research Award in the Medical Sciences.

Competing interests: None declared.

1 Maudsley H. Body and mind. London: Macmillan, 1873.

2 Toone B. Epilepsy. In: Gelder M, Lopez-Ibor J, Andreasen N, eds. The new Oxford textbook of psychiatry. Oxford: Oxford University Press, 2000:1153-7.

3 Fazel S, Danesh J. Serious mental disorder among 23000 prisoners: a systematic review of 62 surveys. Lancet 2002;359:545-50.

4 Sander G, Shorvon S. Epidemiology of the epilepsies. J Neurol Neurosurg Psychiatry 1996;61:433-43.

5 Rowan A, Hyman H, French JH. The prevalence of epilepsy in a large heterogeneous urban population (The Bronx, New York, January 8, 1975). Trans Am Neurol Assoc 1976;101:281-3.

(Accepted 28 February 2002)
Department of

Psychiatry,

University of

Oxford, Warneford

Hospital, Oxford

OX3 7JX

Seena Fazel

research fellow

Evangelos Vassos

senior house officer

Department of

Public Health and

Primary Care,

Institute of Health

Sciences, University

of Cambridge,

Cambridge

CB2 2SR

John Danesh

professor of

epidemiology and

medicine

Correspondence to:

S Fazel

BMJ 2002;324:1495

(1)

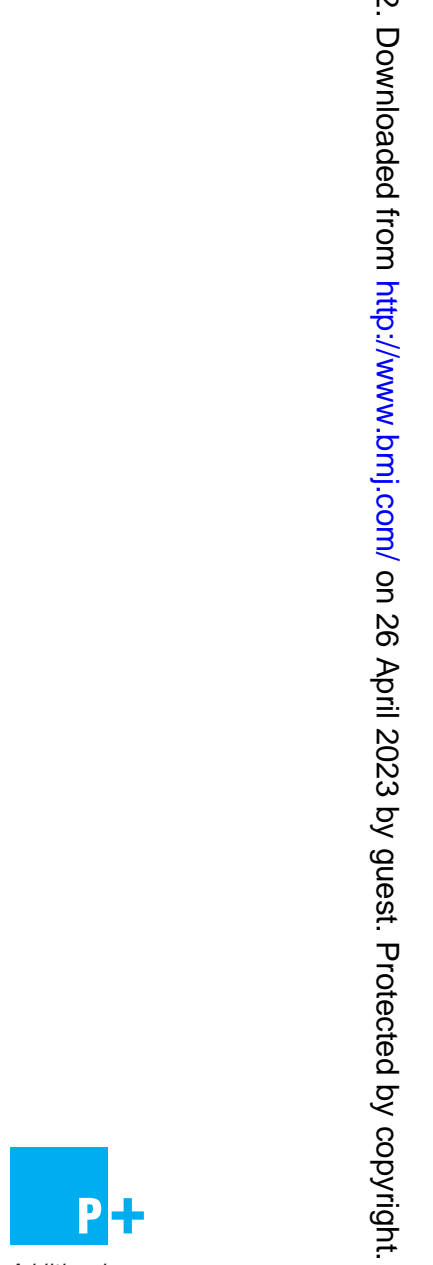

Additional

references appear

on bmj.com 